\title{
Discovery of an activity cycle in the solar analog HD $45184^{\star}$ Exploring Balmer and metallic lines as activity proxy candidates
}

\author{
M. Flores ${ }^{1,5}$, J. F. González ${ }^{1,3,5}$, M. Jaque Arancibia ${ }^{1,5}$, A. Buccino ${ }^{2,4,5}$, and C. Saffe ${ }^{1,3,5}$ \\ 1 Instituto de Ciencias Astronómicas, de la Tierra y del Espacio (ICATE), España Sur 1512, CC 49, 5400 San Juan, Argentina \\ e-mail: [mflores; fgonzalez; mjaque; csaffe]@icate-conicet.gob.ar \\ 2 Instituto de Astronomía y Física del Espacio (IAFE), Buenos Aires, Argentina \\ e-mail: abuccino@iafe.uba.ar \\ 3 Facultad de Ciencias Exactas, Físicas y Naturales, Universidad Nacional de San Juan, 5400 San Juan, Argentina \\ ${ }^{4}$ Departamento de Física, Facultad de Ciencias Exactas y Naturales, Universidad de Buenos Aires, Buenos Aires, Argentina \\ 5 Consejo Nacional de Investigaciones Científicas y Técnicas (CONICET), Argentina
}

Received 16 January 2016 / Accepted 2 April 2016

\section{ABSTRACT}

\begin{abstract}
Context. Most stellar activity cycles similar to that found in the Sun have been detected by using the chromospheric Ca II H\&K lines as stellar activity proxies. However, it is unclear whether such activity cycles can be identified using other optical lines.

Aims. We aim to detect activity cycles in solar-analog stars and determine whether they can be identified through other optical lines, such as Fe II and Balmer lines. We study the solar-analog star HD 45184 using HARPS spectra. The temporal coverage and high quality of the spectra allow us to detect both long- and short-term activity variations.

Methods. We analysed the activity signatures of HD 45184 by using 291 HARPS spectra obtained between 2003 and 2014 . To search for line-core flux variations, we focused on $\mathrm{Ca}$ II $\mathrm{H} \& \mathrm{~K}$ and Balmer $\mathrm{H} \alpha$ and $\mathrm{H} \beta$ lines, which are typically used as optical chromospheric activity indicators. We calculated the HARPS-S index from Ca II H\&K lines and converted it into the Mount Wilson scale. In addition, we also considered the equivalent widths of Balmer lines as activity indicators. Moreover, we analysed the possible variability of Fe II and other metallic lines in the optical spectra. The spectral variations were analysed for periodicity using the Lomb-Scargle periodogram.

Results. We report for the first time a long-term 5.14-yr activity cycle in the solar-analog star HD 45184 derived from Mount Wilson $\mathrm{S}$ index. This makes HD 45184 one of most similar stars to the Sun with a known activity cycle. The variation is also evident in the first lines of the Balmer series, which do not always show a correlation with activity in solar-type stars. Notably, unlike the solar case, we also found that the equivalent widths of the high photospheric Fe II lines (4924 $\AA, 5018 \AA$ and $5169 \AA$ ) are modulated ( \pm 2 m $)$ by the chromospheric cycle of the star. These metallic lines show variations above $4 \sigma$ in the rms spectrum, while some Ba II and Ti II lines present variations at $3 \sigma$ level, which could be considered as marginal variations. From short-term modulation of the $\mathrm{S}$ index we calculate a rotational period of 19.98 days, which agrees with its mean chromospheric activity level. We also clearly show that the activity cycles of HD 45184 can be detected in both Fe II and Balmer lines.
\end{abstract}

Key words. stars: activity - stars: chromospheres - stars: solar-type - stars: individual: HD 45184

\section{Introduction}

The pioneer research on stellar activity by Wilson (1978) and subsequent works (e.g. Vaughan et al. 1978; Duncan et al. 1991; Gray \& Baliunas 1995) have allowed to better understand the activity phenomenon in stars other than the Sun. A main contribution to this subject was made by Baliunas et al. (1998), who analysed the flux variability of Ca II H\&K lines and found three different types of behaviour. They found cycles with periods of between 2.5 and $25 \mathrm{yr}$ that are often associated with stars with moderate activity. Very active stars display fluctuations of activity rather than cycles, while inactive stars seem to be in a state similar to the solar Maunder minimum, the time period between 1645 and 1715, when the Sun deviated from its usual 11-yr activity cycle.

Since the pioneering surveys, several stellar cycles have been reported to date (e.g. Hall et al. 2007; Metcalfe et al. 2010, 2013; DeWarf et al. 2010; Buccino et al. 2014; Egeland et al. 2015).

\footnotetext{
$\star$ Based on data products from observations made with ESO Telescopes at the La Silla Paranal Observatory under programs 072.C-0488 and 183.C-0972.
}

Activity cycles have been identified in stars of spectral types $\mathrm{F}$ to $\mathrm{M}$ (even stars with exoplanets), including stars with multiple cycles. The Ca II H\&K lines are commonly used as optical activity proxies (e.g. Baliunas \& Jastrow 1990; Baliunas et al. 1998; Hall et al. 2007), as are other lines, such as those of the Balmer series (Robertson et al. 2013a; Reiners et al. 2012) or the Mg II infrared triplet (see e.g. Busà et al. 2007; Pietarila \& Livingston 2011). However, it is unclear whether the activity cycles can be identified using other optical lines. These activity cycles have important implications on different fields. For instance, studying a range of stars with physical characteristics similar to the Sun across a range of ages and other parameters is very useful for understanding how typical the Sun is (e.g. Hall et al. 2007, 2009). It also helps in particular to understand the frequency of Earthinfluencing behaviours (e.g. Maunder minimum). The discovery of activity cycles also helps in the important task of distinguishing stellar and planetary signals in radial-velocity surveys (e.g. Robertson et al. 2013b; Carolo et al. 2014).

We started a program aiming to detect activity cycles in solar-analog stars using the extensive database of HARPS 
spectra. Our initial sample comprises close solar-analog stars taken from Nissen (2015), who carefully selected stars with physical parameters very similar ${ }^{1}$ to the Sun $\left( \pm 100 \mathrm{~K}\right.$ in $T_{\text {eff }}$, $\pm 0.15 \mathrm{dex}$ in $\log g$, and $\pm 0.10 \mathrm{dex}$ in $[\mathrm{Fe} / \mathrm{H}]$ ), and we also required that the sample spectra have a very high signal-to-noise ratio $S / N(\geq 600)$. The similarity between these stars and the Sun, together with the possibility to include spectra with high $\mathrm{S} / \mathrm{N}$ and a homogeneous parameter determination, encouraged us to initially select these objects. However, we do not discard the future possible inclusion of more stars to extend our sample.

An inspection of the preliminary data showed a notable variation in the chromospheric activity of G1.5V (Gray et al. 2006) star HD 45184 (=HR 2318, $V=6.39, B-V=0.62)$. It is located at a distance of $\sim 22 \mathrm{pc}$ (van Leeuwen 2007) and hosts a debris disk of 1-2 $M_{\oplus}$ (Lawler et al. 2009). The reported fundamental parameters are $T_{\text {eff }}=5871 \pm 6 \mathrm{~K}, \log g=4.445 \pm 0.012 \mathrm{dex}$, $[\mathrm{Fe} / \mathrm{H}]=0.047 \pm 0.006 \mathrm{dex}$, and $v_{\text {turb }}=1.06 \pm 0.017 \mathrm{~km} \mathrm{~s}^{-1}$ (Nissen 2015). Nissen also derived a mass of $1.06 \pm 0.01 M_{\odot}$ (only internal error) and age of $2.7 \pm 0.5 \mathrm{Gyr}$, showing that HD 45184 is $\sim 1.8$ Gyr younger than the Sun. Then, with the aim of detecting possible activity cycles, we collected all the available HARPS spectra for this object. Multiple observations are required to properly identify both long- and short-term activity variations. For HD 45184, we gathered a total of 291 spectra covering more than ten years of observations, which makes this source a very good target in which to search for a possible activity cycle. HD 45184 has also been identified as a candidate to host a planetary mass companion of $0.04 M_{\text {Jup }}$ that was detected by radial velocity (Mayor et al. 2011), and it is included in the Extrasolar Planets Encyclopaedia ${ }^{2}$. All this makes HD 45184 a very interesting object and encouraged us to perform the study presented here.

This work is organised as follows. In Sect. 2 we describe the observations and data reduction, while in Sect. 3 we describe our results, and finally in Sect. 4 we present our discussion.

\section{Observations and data reduction}

The spectra were obtained from the European Southern Observatory (ESO) archive $^{3}$ under the ESO programs 072.C-0488(E) (PI: M. Mayor and S. Udry) and 183.C-0972(A) (PI: S. Udry). The observations were taken with the HARPS spectrograph attached to the La Silla 3.6 m telescope between 2003 to 2014 and have been automatically processed by the HARPS pipeline ${ }^{4}$. The spectra have a resolving power $R \sim 115000$ and cover the spectral range 3782-6913 $\AA$. After discarding a few low S/N observations, we obtained a total of 291 spectra with a mean $S / N \sim 175$ at $6490 \AA$.

Since this work was intended to search for low-level flux variations, the spectra were carefully normalised and cleaned. Cosmic rays were identified and removed considering a threshold of five times the noise level. Continuum differences between the spectra were corrected for by filtering low-frequency modulations.

\footnotetext{
1 Strictly speaking, this is a sample of solar-analog stars and not of "solar-twins", as they were called by Nissen, because (for instance) the age interval covered was $0.7-8.8 \mathrm{Gyr}$.

2 http://exoplanet.eu/

3 https://www.eso.org/sci/facilities/lasilla/

instruments/harps/tools/archive.html

4 http://www.eso.org/sci/facilities/lasilla/

instruments/harps/doc.html
}

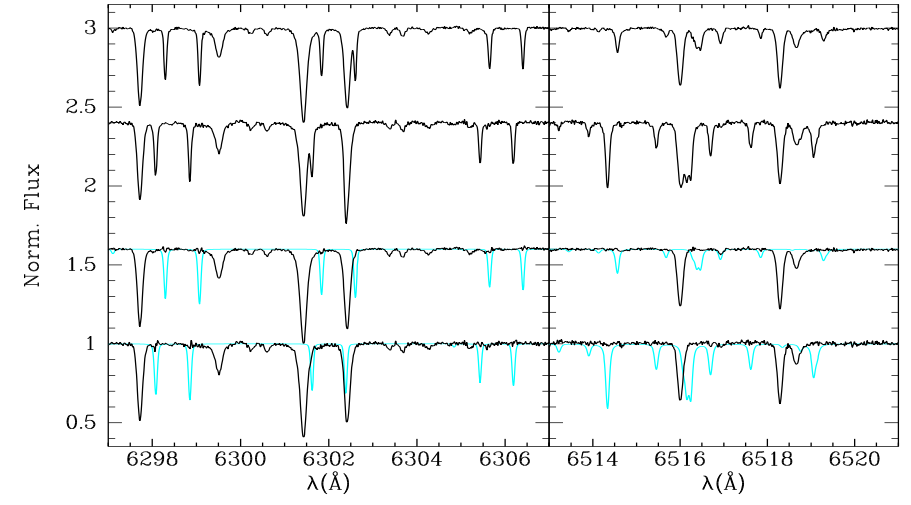

Fig. 1. Removal of telluric lines. For two observations of different runs the original spectra are shown in the top panel. Below the clean spectra (black) are overplotted with the telluric model spectra used in each case (light blue).

Telluric features were removed by dividing each observed spectrum $S_{\text {obs }}$ by an empirical telluric spectrum $T$ appropriately scaled:

$S_{\text {corr }}=S_{\mathrm{obs}} \cdot T^{-\alpha}$,

where $\alpha$ represents the relative optical thickness of the terrestrial atmosphere. This coefficient $\alpha$ was chosen to reproduce the telluric line intensities, most of which are due to water vapour in our spectral region. We repeated this procedure using a template of the $\mathrm{O}_{2} \gamma$-band around $6300 \AA$. In this way, we were able to satisfactorily correct observations taken with different atmospheric water vapour content. Figure 1 shows an example of the removal of telluric lines in two small spectral windows. Telluric lines in the left side of the bottom panel are due to atmospheric $\mathrm{O}_{2}$, while the right side corresponds to $\mathrm{H}_{2} \mathrm{O}$.

\section{Results}

\subsection{Chromospheric Ca II H\&K lines}

The HARPS-S index was obtained following the classical method used to calculate the Mount Wilson $\mathrm{S}$ index (Vaughan et al. 1978). We integrated the flux in two windows centred at the cores of the CaII H\&K lines (3933.664 $\AA$ and $3968.470 \AA$ ), weighting with triangular profiles of $1.09 \AA$ FWHM. The ratio of these fluxes to the mean continuum flux was computed by using two $20 \AA$ wide passbands centred at $3891 \AA$ and $4011 \AA$. Finally, we derived the Mount Wilson $\mathrm{S}$ index by using the calibration procedure explained in Lovis et al. (2011).

In Fig. 2 we plot the time series of the original 291 HARPS measurements. The total uncertainty $\sigma_{\text {tot }}$ of individual S determinations was derived by quadratically adding both the observational error $\sigma_{\text {obs }}$ (obtained as in Henry et al. 1996) and the systematic error associated with the calibration between the HARPS and Mount Wilson activity indexes $\left(\sigma_{\text {sys }} \sim 0.0043\right.$, according to Lovis et al. 2011). For clarity, error bars corresponding to $\sigma_{\text {tot }}$ are not shown in Fig. 2 because they are almost identical for all points. In addition to the individual measurements, we include in the Fig. 2 the average values of observations associated with the same observing season. Error bars of the average values correspond to the standard deviation of the mean. For bins with one measurement, we adopted the typical rms dispersion 


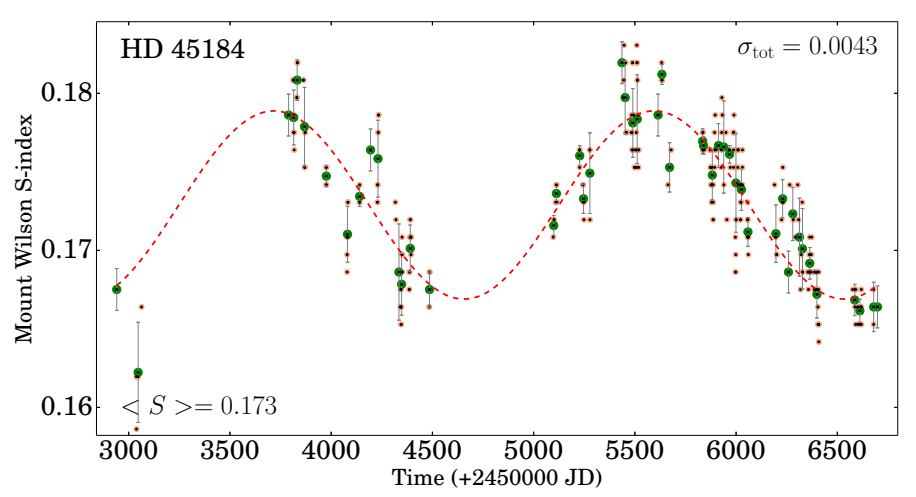

Fig. 2. Time series of Mount Wilson index measurements of HD 45184 from the full HARPS data set (small orange circles) and seasonal means (large green circles). The dotted red line indicates the cycle calculated in this work. The error of individual measurements is probably about 0.0043 .

of other bins. Both time series show clear evidence of a chromospheric activity cycle. By applying the Lomb-Scargle periodogram (Horne \& Baliunas 1986) to the seasonal means, we derived a period $P=1878 \pm 9$ days $(\sim 5.14 \mathrm{yr})$. We used seasonal means in this analysis to reduce the rotational scatter, as is usual (e.g. Baliunas et al. 1995; Gomes da Silva et al. 2011). The false-alarm probability (FAP) corresponding to the main peak is $3.1 \times 10^{-07}$.

Along with the observational data, Fig. 2 shows the harmonic curve of the period of 1878 days (red dotted line), obtained as the least-squares fit to the seasonal means with a confidence level of $99 \%$ (see Buccino \& Mauas 2009, for details). The semiamplitude of the fitted curve is $\Delta S=0.0062 \pm 0.0002$ and the epoch of maximum activity corresponds to JD 2455600.

The mean Mount Wilson index of HD 45184 obtained in this work is $\langle S\rangle=0.173$, which is indistinguishable from the values derived by Henry et al. $(1996,\langle S\rangle=0.173)$ and Gray et al. $(2006,\langle S\rangle=0.172)$. The mean activity level of HD 45184 is therefore similar to that of the Sun $\left(\langle S\rangle_{\odot}=0.171\right.$, Hall et al. 2009).

\subsection{Balmer and metallic lines as activity indicators}

Several studies have suggested that the correlation between the chromospheric emission from the $\mathrm{Ca}$ II $\mathrm{H} \& \mathrm{~K}$ and $\mathrm{H} \alpha$ lines present in Sun (Livingston et al. 2007) might be found in other stars (e.g. Meunier \& Delfosse 2009; Martínez-Arnáiz et al. 2010, 2011; Stelzer et al. 2013; Gomes da Silva et al. 2014). Even though this correlation has been reported as not always valid (Cincunegui et al. 2007), the activity in the Sun and other stars measured by $\mathrm{Ca}$ II $\mathrm{H} \& \mathrm{~K}$ emission might correlate with other chromospheric and even high photospheric lines (e.g. Hall \& Lockwood 1998; Livingston et al. 2007).

To search for such correlations, we looked for possible variations in $\mathrm{H} \mathrm{I}$ and metallic lines in the spectra using different techniques. On the one hand, we averaged the 291 spectra and built an rms spectrum by calculating the residual rms at each wavelength. Before these calculations, a mild filter in low and high frequencies was applied to the residual spectra to remove continuum differences and reduce shot noise. Since shot noise is an important contribution to the observed rms, strong lines in general present a rms value lower than the surroundings. For this reason, we divided the rms spectrum by the theoretical noiselevel spectrum calculated from photon statistics.
As a second strategy, we applied the technique of Sokolov (2000). In this case, all the flux measurements at a given wavelength are used to produce a light curve that is fitted by leastsquares with the first terms of a Fourier series. We fixed the period and derived the amplitude of the flux variation at each wavelength assuming a sinusoidal function.

From this analysis we detected significant variations in addition to those of the Ca II lines near the core of the H I lines and in some strong iron lines, particularly Fe II lines at $4924 \AA$, $5018 \AA$, and $5169 \AA$. Figure 3 shows the rms spectrum and the light-variation amplitude spectrum for small spectral windows around selected lines. The amplitude and rms spectra were normalised to the mean noise level in the region. All mentioned lines (Ca II H\&K, H $\beta$, H $\alpha$, Fe II $4924 \AA$, $5018 \AA$, and $5169 \AA$ ) present variations above $4 \sigma$, with flux variations of about $0.6 \%$ of the continuum level. Additionally, a few other lines of ionised metals exhibit variations at a $3 \sigma$ level, which we consider here as marginal detections. These lines are the Ba II line at $6141.8 \AA$ and Ti II lines at $4395.0 \AA, 4443.8 \AA, 4468.5 \AA, 4501.3 \AA$, and $4572.0 \AA$. In these cases, flux variations are about $0.4-0.5 \%$ of the continuum.

To study the temporal behaviour, we derived equivalent width variations by integrating the residual spectra around the central wavelength of the variable lines. Figure 4 shows the Mount Wilson S index along with the equivalent width of $\mathrm{HI}$ and Fe II lines as a function of the activity cycle phase. There is a clear correlation between the intensity of the lines and the chromospheric activity for the lines $\mathrm{H} \beta$, Fe II $4924 \AA$, and Fe II $5018 \AA$. For $\mathrm{H} \alpha$, even though the spectral variability of the line core ( $\pm 1 \AA$ around the line centre) is beyond doubt, no noticeable net changes are observed in the equivalent width. This is evident in Fig. 5, where we compare the average of 85 residual spectra close to the low activity phase (blue) and the average of 57 spectra around the activity maximum (red). To quantify the spectral variations in $\mathrm{H} \alpha$, we calculated the kurtosis of the line core in an spectral window of $4 \AA$. The resulting values show the same behaviour as other activity indicators, as shown in the second panel of Fig. 4. This shows that Balmer and Fe II line variations reproduce the $5.14 \mathrm{yr}$ activity cycle detected with the Mount Wilson index, although with a lower amplitude-toerror ratio. This is confirmed by the corresponding periodograms (Fig. 6) in which all the highest peaks lie between 1877 and 1879 days (the Mount Wilson indexes, the kurtosis of $\mathrm{H} \alpha$, and the flux of $\mathrm{H} \beta$ and Fe II lines).

\subsection{Rotation period}

As is often the case in other magnetic stars, the point spread in the chromospheric activity curve is significantly larger than the estimated measurement errors, which can be attributed to rotational modulations caused by the passage of individual active regions (Gray \& Baliunas 1995; Brown et al. 2008; Metcalfe et al. 2010). To detect such variations and to determine the rotation period, we followed a strategy similar to that reported by Metcalfe et al. (2010). We subtracted from the individual S index measurements the fitted harmonic curve (red dotted line in Fig. 2) and analysed the periodicity of the residuals with both the Lomb-Scargle periodogram and the phase dispersion minimisation technique (Stellingwerf 1978).

As a result, we found a period of $19.98 \pm 0.02$ days with a very low $\mathrm{FAP}=1.04 \times 10^{-11}$ for the $\mathrm{S}$ index. This analysis was based on CaII lines. The $\mathrm{S} / \mathrm{N}$ of the variations found in other lines is too low to be used for the rotational analysis. 

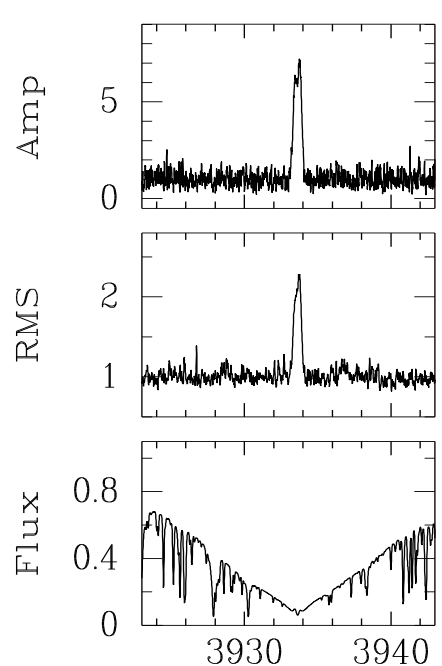

$\lambda(\AA)$
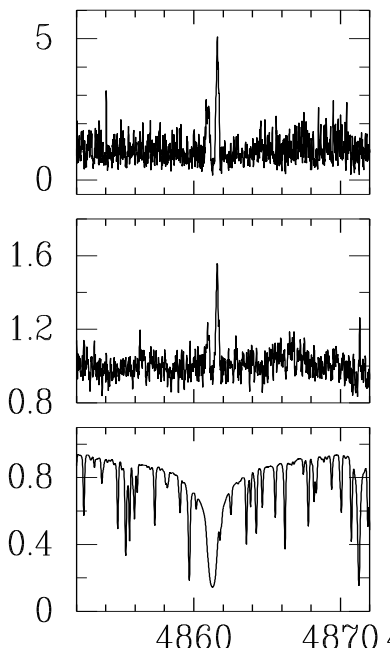

$\lambda(\AA)$
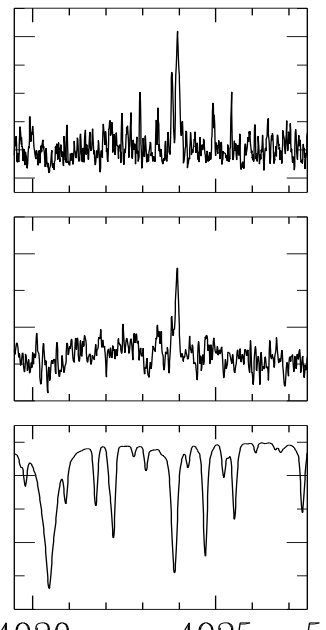

$\lambda(\AA)$
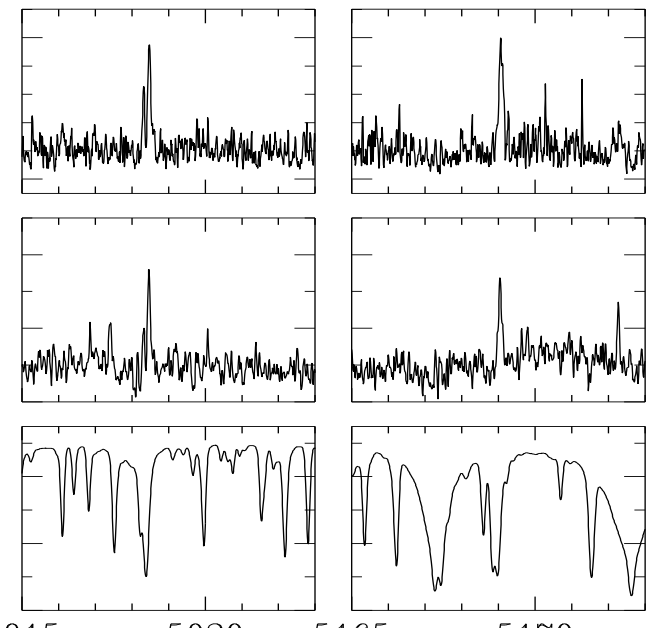

5015

5020

5165

$\lambda(\AA)$

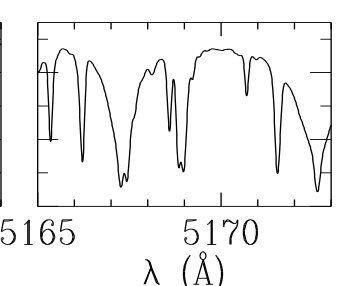

$\lambda(\AA)$

Fig. 3. Spectral variability. Light-variation amplitude spectrum (upper panels) and rms spectrum (middle panels) are shown for a small spectral windows around five variable spectral lines: Ca II 3933, Fe II 4924, Fe II 5018, and Fe II 5169. Lower panels: average spectrum in each region.
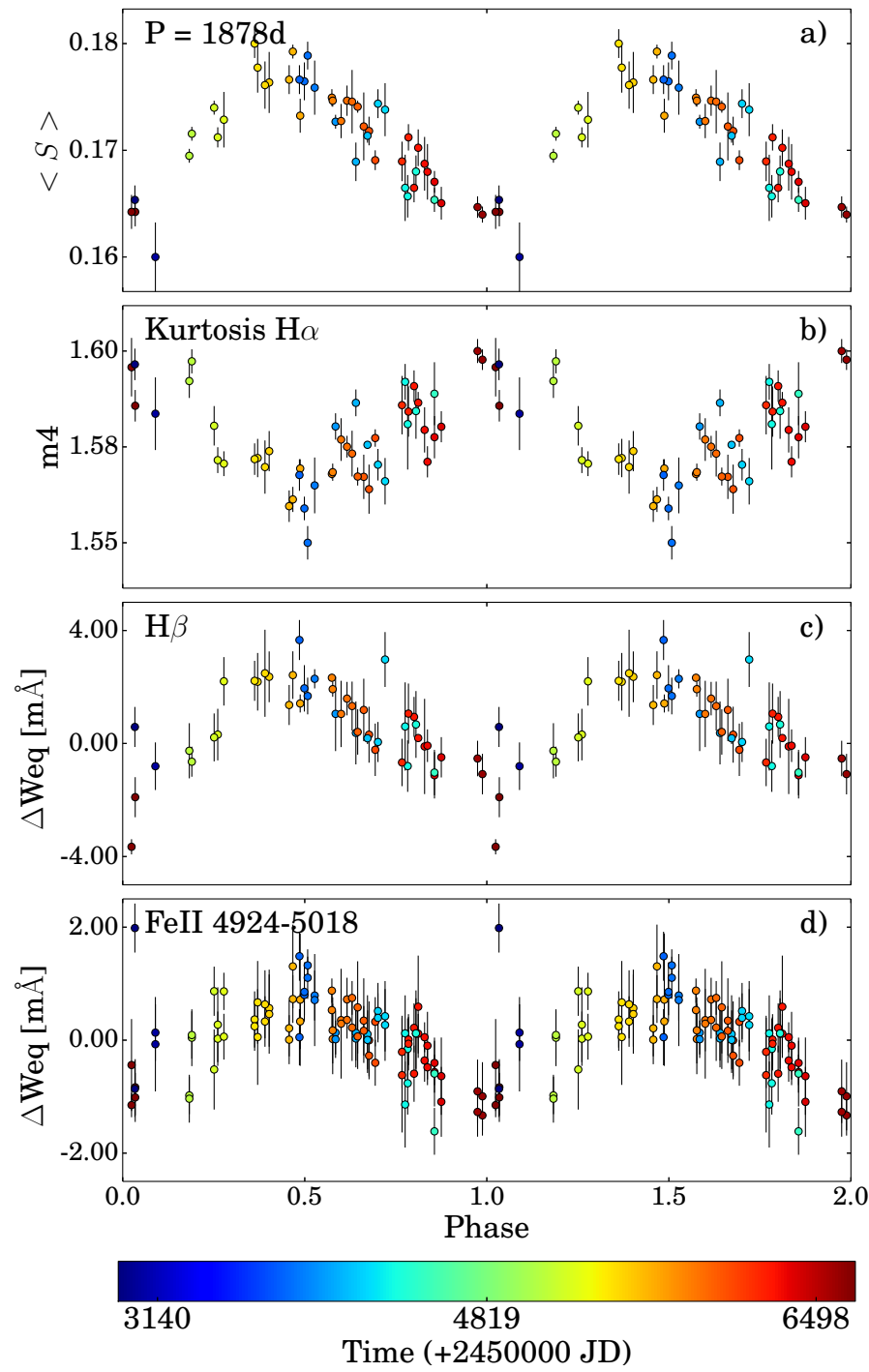

Fig. 4. a) Chromospheric activity measurements from Ca II H\&K lines. b) Kurtosis of the $\mathrm{H} \alpha$ line. Panels c) and d) correspond to the equivalent width of the Balmer H $\beta$, Fe II $4924 \AA$, and Fe II $5018 \AA$ lines. Phases have been calculated with a period of 1878 days.

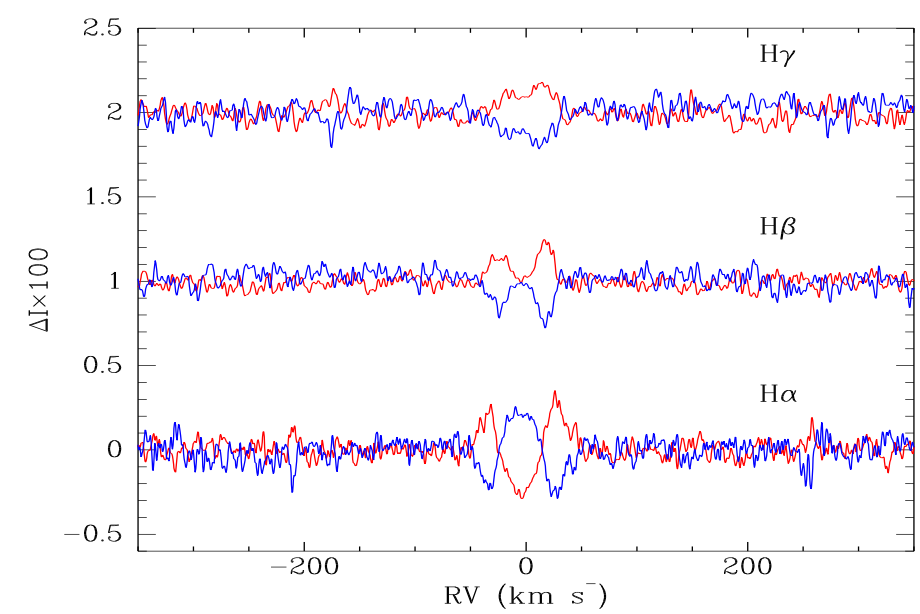

Fig. 5. Difference of Balmer line profiles for high (blue) and low (red) activity phases.

Figure 7 shows the periodogram and the S-index residuals as a function of phase. Our result agrees with the period estimated by Wright et al. (2004; 21 days) using the empirical relation between rotation and chromospheric activity calibrated by Noyes et al. (1984).

\section{Discussion}

We studied the long-term activity of the solar-analog star HD 45184 by using the CaII H\&K and Balmer lines as stellar activity proxies. We analysed the $\mathrm{Ca}$ II $\mathrm{H} \& \mathrm{~K}$ line-core fluxes in 291 HARPS spectra taken between 2003 and 2014. In addition, we detected spectral variability in the first three lines of the Balmer series and in some strong lines of ionised metals, particularly Fe II lines at $4924 \AA$, $5018 \AA$, and $5169 \AA$. We detected a long-term activity cycle of $5.14 \mathrm{yr}$ in the CaII H\&K lines, which is clearly replicated by line profile variations in Balmer and strong Fe II lines (see e.g. Fig. 4). Equivalent width variations of Balmer (except for $\mathrm{H} \alpha$ ) and $\mathrm{Fe}$ II lines present a positive correlation with the emission in the $\mathrm{Ca}$ II $\mathrm{H} \& \mathrm{~K}$ lines: the more intense the emission of the Ca II H\&K lines, the stronger the H I and $\mathrm{Fe}$ II lines. For $\mathrm{H} \alpha$, the equivalent width does not change 
M. Flores et al.: Discovery of an activity cycle in the solar analog HD 45184

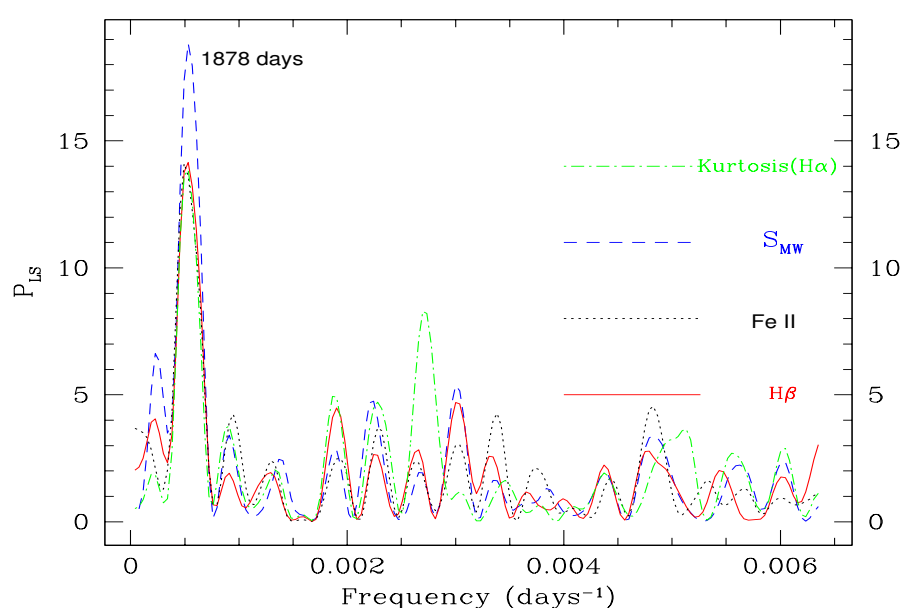

Fig. 6. Lomb-Scargle periodogram from the different line variations plotted in Fig. 4.
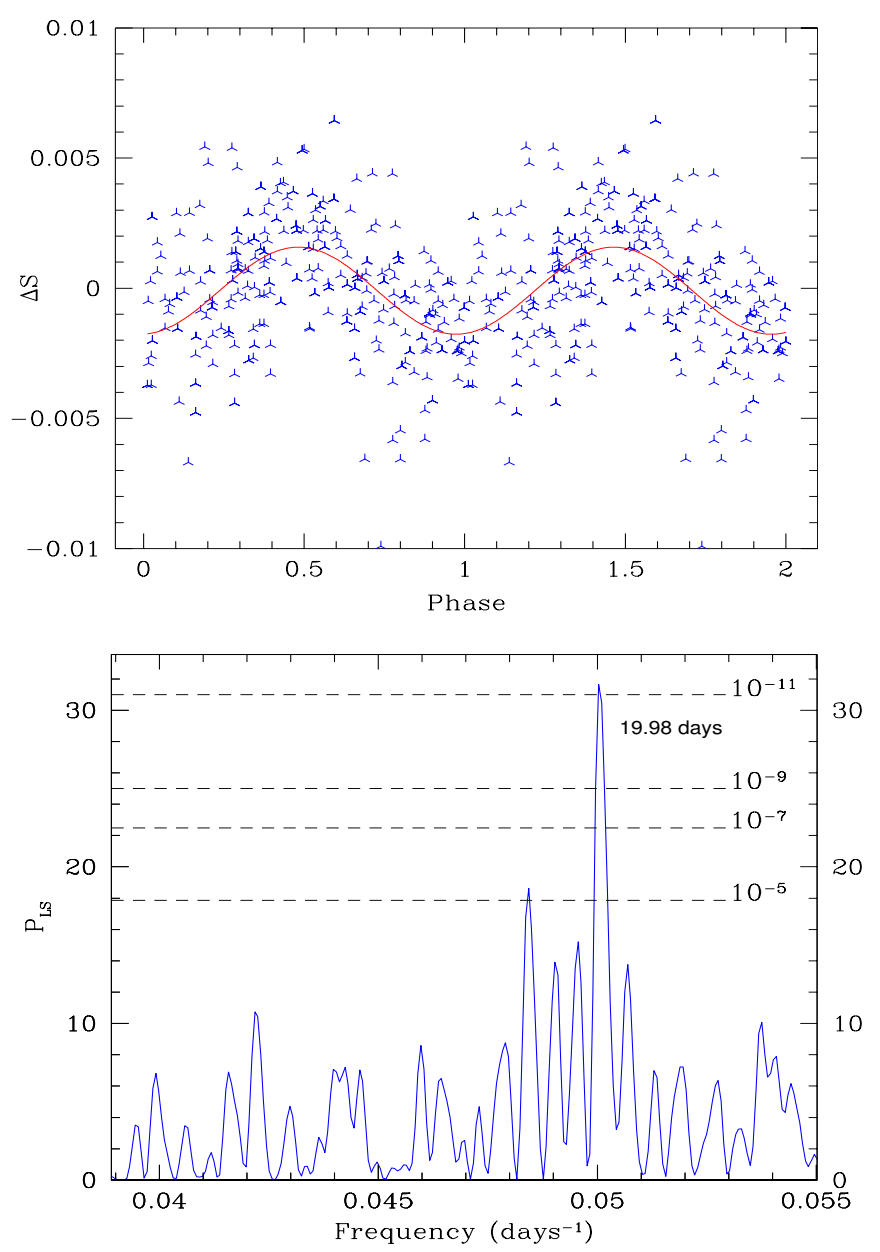

Fig. 7. Top panel: Mount Wilson index residuals phased with a period of $\sim 19.98$ days. Lower panel: Lomb-Scargle periodogram from filtered (residuals) Ca II H\&K measurements. The highest peak suggests a rotation period of 19.98 days.

significantly, although the line profile shape varies in phase with the activity cycle.

It is believed that the surface of older and less active stars, including our Sun, are dominated by bright regions called faculae, whereas young and active stars are spot dominated (Radick et al. 1998; Berdyugina et al. 2002). Moore et al. (1966) showed a number of Fe lines sensitive to the presence of sunspots. Based

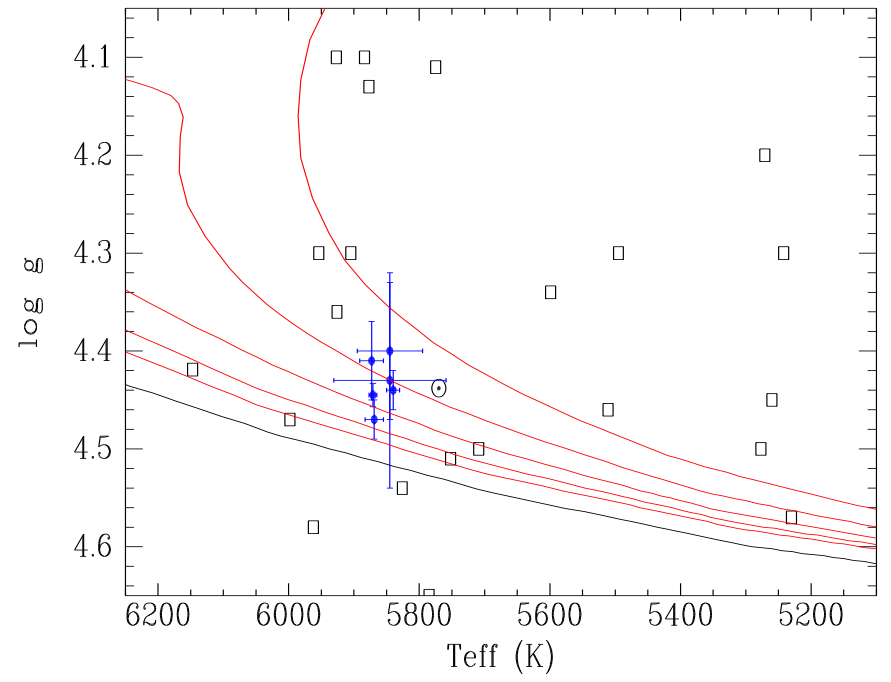

Fig. 8. Position of G-type stars with activity cycles in the $T_{\text {eff }}-\log g$ diagram (black empty squares). Blue filled circles with error bars correspond to the parameters of HD 45184 derived by different authors. Red lines are isochrones for solar abundances shown for log age $=9.0,9.2$, 9.4, 9.6, and 9.8; the black line is the ZAMS. The position of the Sun is also included in the figure.

on this scenario and considering the similarities between the Sun and HD 45184, an Fe II variation following the Ca H\&K activity cycle might be attributed to the younger age of HD 45184. This may be verified from future observations of other young solaranalog stars.

Solar spectral variation of several Fraunhofer lines along the $11 \mathrm{yr}$ activity cycle were first revealed by Livingston \& Holweger (1982). Next, taking into account that these lines are formed at different depths in the atmosphere of the Sun, Livingston et al. (2007) used some photospheric and chromospheric lines to study the long-term variations of the temperature. Monitoring high photospheric lines from 1980 to 2006, the authors found that Mn $5394 \AA$ is the only photospheric line that shows a clear modulation with the chromospheric $11 \mathrm{yr}$ Ca II K cycle of the Sun. Surprisingly, the Fe lines did not show the expected modulation with $\mathrm{Ca}$ II $\mathrm{H} \& \mathrm{~K}$ lines, and therefore the authors suggested that these lines might be following the $22 \mathrm{yr}$ Hale cycle. Interestingly, in HD 45184 we found that Fe II lines show a clear modulation with the 5.14 yr Ca II H\&K activity cycle. Following Moore et al. (1966), the variations of these high photospheric Fe II lines might point toward the possible presence of dark spots on the surface of HD 45184. At the same time and similar to the solar case, the faculae are presumably present on the stellar surface, as suggested by the Ca II H\&K emission lines (e.g. Hall 2008, and references therein).

Using the Ca II H\&K lines, we found for short-term modulations caused by stellar rotation and determined a rotation period of 19.98 days for HD 45184. This value agrees with the rotational period vs. chromospheric activity calibration of Wright et al. (2004). On the other hand, because HD 45184 is more active than the Sun, the rotation period calculated by us is expected to be shorter than the rotation period of the Sun because of the widely assumed close connection between rotation and activity (Noyes et al. 1984).

Although solar-twin and solar-analog stars are ideal laboratories in which to carry out comparative studies of stellar cycles and the solar cycle, only a few have been considered as targets of activity studies. An interesting aspect of HD 45184 is 
that of the stars with detected activity cycles, it is one of those whose stellar parameters are closest to the solar values. In Fig. 8 we show the position in the $T_{\text {eff }}-\log g$ diagram of stars with known activity cycles. We have included G-type stars studied by Choi et al. (2015) and the Mount Wilson project stars that were selected by Schröder et al. (2013) as having cyclic variations. We also added individual objects with activity periods reported by Metcalfe et al. (2010), DeWarf et al. (2010), and Egeland et al. (2015). We also included the parameters of HD 45184 that were determined by different literature works (Sousa et al. 2008; Bensby et al. 2014; Battistini \& Bensby 2015; Maldonado et al. 2015; Nissen 2015; Spina et al. 2016). These are shown as blue filled circles with error bars. For comparison, the theoretical isochrones of Mowlavi et al. (2012) for solar abundances are plotted. HD 45184 has almost the same surface gravity as the Sun within the uncertainties, and it is slightly hotter. In particular, Bensby et al. (2014) estimated an age of 4.4 \pm 2.2 Gyr, which is similar to the age of the Sun. However, the parameters reported by Sousa et al. (2008) suggest that HD 45184 is about 2 Gyr younger than the Sun, in agreement with Nissen (2015). Without going into further detail, we can say that HD 45184 is $\sim 100 \mathrm{~K}$ hotter than the Sun and is one of the closest solar-analog stars with a known activity cycle.

It would be interesting to study whether the Fe II lines might also be used, for instance, to detect a possible activity cycle in those planet-search radial-velocity surveys that do not include the classical Ca II H\&K lines in their spectra. This is the case, for example, of the Anglo-Australian Planet Search survey (e.g. Butler et al. 1996, 2001; Tinney et al. 2011). We caution, however, that more observations are needed to verify the usefulness of the Fe and possibly other metallic lines in solar-analog stars.

Acknowledgements. We thank the anonymous referee for constructive comments that improved the paper.

\section{References}

Baliunas, S., \& Jastrow, R. 1990, Nature, 348, 520

Baliunas, S. L., Donahue, R. A., Soon, W. H., et al. 1995, ApJ, 438, 269

Baliunas, S. L., Donahue, R. A., Soon, W., \& Henry, G. W. 1998, in Cool Stars, Stellar Systems, and the Sun, eds. R. A. Donahue, \& J. A. Bookbinder, ASP Conf. Ser., 154, 153

Battistini, C., \& Bensby, T. 2015, A\&A, 577, A9

Bensby, T., Feltzing, S., \& Oey, M. S. 2014, A\&A, 562, A71

Berdyugina, S. V., Pelt, J., \& Tuominen, I. 2002, A\&A, 394, 505

Brown, K. I. T., Gray, D. F., \& Baliunas, S. L. 2008, ApJ, 679, 1531

Buccino, A. P., \& Mauas, P. J. D. 2009, A\&A, 495, 287

Buccino, A. P., Petrucci, R., Jofré, E., \& Mauas, P. J. D. 2014, ApJ, 781, L9

Busà, I., Aznar Cuadrado, R., Terranegra, L., Andretta, V., \& Gomez, M. T. 2007, A\&A, 466, 1089

Butler, R. P., Marcy, G. W., Williams, E., et al. 1996, PASP, 108, 500

Butler, R. P., Tinney, C. G., Marcy, G. W., et al. 2001, ApJ, 555, 410

Carolo, E., Desidera, S., Gratton, R., et al. 2014, A\&A, 567, A48
Choi, H., Lee, J., Oh, S., et al. 2015, ApJ, 802, 67

Cincunegui, C., Díaz, R., \& Mauas, P. 2007, A\&A, 469, 309

DeWarf, L. E., Datin, K. M., \& Guinan, E. F. 2010, ApJ, 722, 343

Duncan, D. K., Vaughan, A. H., Wilson, O. C., et al. 1991, ApJS, 76, 383

Egeland, R., Metcalfe, T. S., Hall, J. C., \& Henry, G. W. 2015, ApJ, 812, 12

Gomes da Silva, J., Santos, N. C., Bonfils, X., et al. 2011, A\&A, 534, A30

Gomes da Silva, J., Santos, N. C., Boisse, I., Dumusque, X., \& Lovis, C. 2014, A\&A, 566, A66

Gray, D. F., \& Baliunas, S. L. 1995, ApJ, 441, 436

Gray, R. O., Corbally, C. J., Garrison, R. F., et al. 2006, AJ, 132, 161

Hall, J. C. 2008, Liv. Rev. Sol. Phys., 5, 2

Hall, J. C., \& Lockwood, G. W. 1998, ApJ, 493, 494

Hall, J. C., Lockwood, G. W., \& Skiff, B. A. 2007, AJ, 133, 862

Hall, J. C., Henry, G. W., \& Lockwood, G. W. 2009, AJ, 138, 312

Henry, T. J., Soderblom, D. R., Donahue, R. A., \& Baliunas, S. L. 1996, AJ, 111, 439

Horne, J. H., \& Baliunas, S. L. 1986, ApJ, 302, 757

Lawler, S. M., Beichman, C. A., Bryden, G., et al. 2009, ApJ, 705, 89

Livingston, W., \& Holweger, H. 1982, ApJ, 252, 375

Livingston, W., Wallace, L., White, O. R., \& Giampapa, M. S. 2007, ApJ, 657, 1137

Lovis, C., Dumusque, X., Santos, N. C., et al. 2011, A\&A, submitted [arXiv: 1107.5325]

Maldonado, J., Eiroa, C., Villaver, E., Montesinos, B., \& Mora, A. 2015, A\&A, 579, A20

Martínez-Arnáiz, R., Maldonado, J., Montes, D., Eiroa, C., \& Montesinos, B. 2010, A\&A, 520, A79

Martínez-Arnáiz, R., López-Santiago, J., Crespo-Chacón, I., \& Montes, D. 2011, MNRAS, 414, 2629

Mayor, M., Marmier, M., Lovis, C., et al. 2011, A\&A, submitted [arXiv: 1109.2497]

Metcalfe, T., Basu, S., Henry, T. J., et al. 2010, ApJ, 723, L213

Metcalfe, T., Buccino, A., Brown, B., et al. 2013, ApJ, 763, L26

Meunier, N., \& Delfosse, X. 2009, A\&A, 501, 1103

Moore, C. E., Minnaert, M. G. J., \& Houtgast, J. 1966, The solar spectrum $2935 \AA-8770 \AA$ (Washington: US Government Printing Office)

Mowlavi, N., Eggenberger, P., Meynet, G., et al. 2012, A\&A, 541, A41

Nissen, P. E. 2015, A\&A, 579, A52

Noyes, R. W., Hartmann, L. W., Baliunas, S. L., Duncan, D. K., \& Vaughan, A. H. 1984, ApJ, 279, 763

Pietarila, A., \& Livingston, W. 2011, ApJ, 736, 114

Radick, R. R., Lockwood, G. W., Skiff, B. A., \& Baliunas, S. L. 1998, ApJS, 118,239

Reiners, A., Joshi, N., \& Goldman, B. 2012, AJ, 143, 93

Robertson, P., Endl, M., Cochran, W. D., \& Dodson-Robinson, S. E. 2013a, ApJ, 764, 3

Robertson, P., Endl, M., Cochran, W. D., MacQueen, P. J., \& Boss, A. P. 2013b, ApJ, 774, 147

Schröder, K.-P., Mittag, M., Hempelmann, A., González-Pérez, J. N., \& Schmitt, J. H. M. M. 2013, A\&A, 554, A50

Sokolov, N. A. 2000, A\&A, 353, 707

Sousa, S. G., Santos, N. C., Mayor, M., et al. 2008, A\&A, 487, 373

Spina, L., Meléndez, J., \& Ramírez, I. 2016, A\&A, 585, A152

Stellingwerf, R. F. 1978, ApJ, 224, 953

Stelzer, B., Frasca, A., Alcalá, J. M., et al. 2013, A\&A, 558, A141

Tinney, C. G., Butler, R. P., Jones, H. R. A., et al. 2011, ApJ, 727, 103

van Leeuwen, F. 2007, A\&A, 474, 653

Vaughan, A. H., Preston, G. W., \& Wilson, O. C. 1978, MNRAS, 90, 267

Wilson, O. C. 1978, ApJ, 226, 379

Wright, J. T., Marcy, G. W., Butler, R. P., \& Vogt, S. S. 2004, ApJS, 152, 261 